\title{
Effect of Training Systems on Accumulation of Flavan-3-ols in Cabernet Sauvignon Grape Seeds at the North Foot of Mt. Tianshan
}

\author{
Y. Liu ${ }^{1,2}$, J. Yan ${ }^{3}$, Q. Li ${ }^{4}$ J. Wang ${ }^{1,2}$, Y. Shi ${ }^{1,2^{*}}$
}

(1) Centre for Viticulture \& Oenology, College of Food Science and Nutritional Engineering, China Agricultural University, Beijing 100083, China

(2) Key Laboratory of Viticulture and Oenology, Ministry of Agriculture, Beijing 100083

(3) Zhengzhou Fruit Research Institute, Chinese Academy of Agricultural Sciences, Zhengzhou 450000, China

(4) Vegetable and Flower Research Institute, Chinese Academy of Agricultural Sciences, Beijing, 100081, China

Submitted for publication: February 2017

Accepted for publication: November 2017

Keywords: Training systems, flavan-3-ols, seeds, Cabernet Sauvignon

The effect of training system on the content and composition of flavan-3-ols in seeds throughout the development of Cabernet Sauvignon grapes during two consecutive seasons (2011 to 2012) in the northwest of China was evaluated. The applied training systems include the modified vertical shoot positioned (M-VSP); the fan training system with two trunks (F-TT); and the fan training system with multiple trunks (F-MT). The results show that the pattern of flavan-3-ol accumulation was similar for the three training systems in both vintages, with flavan-3-ols being the highest around véraison, before declining as harvest approaches. Compared to the 2011 vintage, the dry vintage of 2012 had a greater accumulation of total flavan-3-ols and individual flavan-3-ol monomers, including (+)-catechin (C), (-)-epicatechin (EC) and (-)-epicatechin-3-O-gallate (ECG). With regard to the effect of training systems, M-VSP contributed to a significantly higher content of total flavan-3-ols, while the percentage of ECG was lower for M-VSP. As for the three flavan-3-ol units (terminal subunits, extension subunits and monomers), the 2012 vintage was more likely to accumulate extension subunits and monomers, and the effect of training system on these three flavan-3-ols units varied between the different vintages. Meanwhile, the proportion of ECG in terminal subunits and of $C$ in extension subunits was higher in 2012, whereas the training system of F-TT had a higher percentage of ECG in extension subunits in both vintages. In addition, the effect of vintage on the composition of flavan-3-ols in harvested grape seeds was more obvious than that of the training system.

\section{INTRODUCTION}

Flavan-3-ols are responsible for the organoleptic characteristics and biochemical properties of wine. Through the formation of complexes with salivary proteins, flavan3 -ols provide red wine with astringency and mouthfeel characteristics (Casassa \& Harbertson, 2014). During wine ageing, flavan-3-ols can also take part in condensation reactions with anthocyanins to stabilise wine colour (Alcalde-Eon et al., 2006; Fulcrand et al., 2006). It is well known that moderate consumption of wine enhances its health benefits, and one of the most important contributors is flavan-3-ols. They possess a number of health-enhancing characteristics, like antioxidants and free radical scavengers, anti-carcinogenics, cardiopreventive and antimicrobial activities, and so on (Renaud \& De Lorgeril, 1992; Aron \& Kennedy, 2008).

Among the phenolic compounds, flavan-3-ols are the most common group of flavonoids. The flavan-3-ols observed in grape seeds are mainly represented by (+)-catechin (C), (-)-epicatechin (EC) and (-)-epicatechin-3-O-gallate (ECG). Proanthocyanidins, as the polymers of flavan-3-ols, are composed of terminal and extension subunits analogous to the flavan-3-ols C, EC and ECG. Subunits are linked via interflavan bonds between C-4 and C-8, and less commonly between C-4 and C-6 (Haslam, 1998). The content and presence of flavan-3-ols in grape seeds depend mainly on variety, soil, climate and viticulture factors, among others. Furthermore, due to a great amount of extraction of flavan3 -ols from grape seeds during the red winemaking process (Sun et al., 1999), their eventual concentration and ability to act as astringents in red wine are affected correspondingly (Ough \& Nagaoka, 1984; Morrison \& Noble, 1990; González-Neves et al., 2002; Kemp et al., 2011). The extent of bitterness and astringency of wine depends largely on the composition of flavan-3-ols and the degree of polymerisation of proanthocyanidins. It has been reported that a higher proportion of ECG supplies wine with a coarse perception, 
while epigallocatechin (EGC) flavan-3-ol monomer, which is only found in skins, smooths the taste (Vidal et al., 2003). In addition, the extent of astringency increases with the degree of polymerisation of proanthocyanidins (Peleg et al., 1999; Maury et al., 2001), of which the value found in seeds is always lower than that in the skins (Downey et al., 2003). This may be the reason why oenologists prefer flavan-3-ols from the seeds and not from the skins.

The modification of the vine training system can optimise the microclimatic conditions of grapes, such as temperature, humidity, sunlight exposure and other environmental factors, which have a direct impact on fruit composition (Gladstone \& Dokoozlian, 2003; Reynolds \& Heuvel, 2009). The impact of grapevine training system on the chemical composition of grapes and wine has been studied widely. Zoecklein et al. (2008) showed that the training system had no significant effect on berry weight, $\mathrm{pH}$ and titratable acidity of 'Viognier' grapes. A similar result was found in relation to 'Niagara' grapes (Sabbatini et al., 2015). However, the concentrations of volatile compounds and phenolic compounds found in grapes and wine were significantly affected by the training system. Compared with four rays (FR), the training systems of little tree (LT) and bilateral Guyot (BG) tended to accumulate aroma precursors and volatile compounds (Fragasso et al., 2012). With regard to the effect of training system on anthocyanins, vertical shoot-positioned (VSP) vines contributed to significantly higher levels of total individual anthocyanins in grapes compared with single Guyot (SG) and four-arm Kniffin (4AK) (Liu et al., 2015). In addition, the concentration of anthocyanins was further investigated in wine, and it was found that an appropriate training system was beneficial for colour stability (Pérez-Lamela et al., 2007; Segade et al., 2009). Furthermore, in a study of the effect of four training systems on 'Frontenac' grapes in south-eastern Nebraska, a higher total phenol content was found with VSP (Bavougian et al., 2013). To the best of our knowledge, the pattern of flavan-3-ol accumulation from fruit set to harvest, in both skin and seeds, has been discussed intensively in previous research (Santos-Buelga et al., 1995; De Freitas \& Glories, 1999; Kennedy et al., 2000a; Downey et al., 2003; Hanlin \& Downey, 2009). However, the influence of training system on the composition of flavan-3-ols has never been studied.

The modified vertical shoot positioned (M-VSP) training system, the fan training system with two trunks (F-TT), and the fan training system with multiple trunks (F-MT) referred to in this study have been applied in the northwest of China. However, there is no published information about the effect of these training systems on the content and composition of flavan-3-ols in grape skins and seeds. This is particularly significant because of the important role flavan-3-ols play in wine quality. Considering this, the aim of the present work was to enlarge our understanding of the effect of these three training systems on the accumulation of flavan-3-ols in grape seeds. This study was performed in a commercial vineyard of Vitis vinifera L. cv Cabernet Sauvignon during two consecutive seasons in the hope that this would provide information for the production of high-quality wine in this region.

\section{MATERIALS AND METHODS}

\section{Reagents and chemicals}

Standards for C, EC, EGC, ECG and phloroglucinol were supplied by Sigma-Aldrich Co. (St. Louis, MO, USA). HPLC-grade acetonitrile, acetone, methanol and acetic acid were purchased from the Fisher Company (Fairlawn, NJ, USA); acetone, ascorbic acid, sodium acetate and $\mathrm{HCl}$ were obtained from the Beijing Chemical Reagent Plant (Beijing, China); and deionised water $(<18 \mathrm{M} \Omega$ resistance) was acquired using a Milli-Q Element water purification system (Millipore, Bedford, MA, USA).

\section{The experimental site and plant materials}

The field experiments were conducted in a commercial, furrow-irrigated vineyard (Yuanyi Farm) during the 2011 and 2012 vintages. Yuanyi Farm is located at the northern foot of Mt. Tianshan, which has an altitude of $475 \mathrm{~m}$ and is

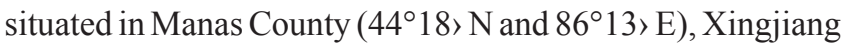
Province, China. The Cabernet Sauvignon grapevines were planted in 2000, own rooted, and arranged in a North-South row orientation. The soil of the vineyard is "silt loam", with $42 \%$ sand in the topsoil (Cheng et al., 2014). The vines were managed for nutrition and pest management according to official industry standards established by the China Supervision Bureau (GB 12696-1990, GB/T 25393-2010).

In this vineyard, the vines were trained according to three training systems (modified VSP, and fan-shaped systems with two trunks and multiple trunks) in 2009 (Fig. 1). The vines were trained on a sloped trunk with a horizontal spurpruned cordon at $0.7 \mathrm{~m}$ above the ground and were spaced at $2.5 \mathrm{~m} \times 1.0 \mathrm{~m}$, which we called modified VSP (M-VSP). Shoots in this system were positioned vertically upright with the aid of wires. The F-TT and F-MT training systems are two kinds of fan-shaped systems with two trunks (F-TT) and multiple trunks (F-MT) respectively. The shoots of the fan training systems face multiple directions. Three training systems were imposed in a completely randomised design with three biological replicates on 45 vines, equally arranged in three consecutive rows. Harvest dates were determined by the cooperating winery. All the grape samples were harvested at technological ripeness, on 2011-09-23 and 2012-09-24.

\section{Berry sampling and general analytical parameters}

Three hundred berries per experimental unit were randomly collected for each replicate, from different positions within the clusters and vines. Véraison began on 2011-07-30 and 2012-07-26 respectively, and lasted for about two weeks. Sampling took place at 31 days after flowering (DAF) (0601), 45 DAF (07-15), 60 DAF (07-30), 66 DAF (08-05), 77 DAF (08-16), 94 DAF (09-02) and 115 DAF (09-23) in 2011 and at 32 DAF (07-02), 46 DAF (07-16), 56 DAF (07-26), 68 DAF (08-08), 75 DAF (08-15), 89 DAF (08-29) and 115 DAF (09-24) in 2012. The samples were frozen and conserved at $-80^{\circ} \mathrm{C}$.

One hundred and 50 berries (50 per replicate) were used for the determination of berry weight, and were crushed in a hand press to obtain juice. The total soluble solids (TSS) of the juice were measured using a PAL-1 Digital Hand-held "Pocket" Refractometer (Atago, Tokyo, Japan). Titratable acidity (TA) was measured by titration with $\mathrm{NaOH}$ to the end 

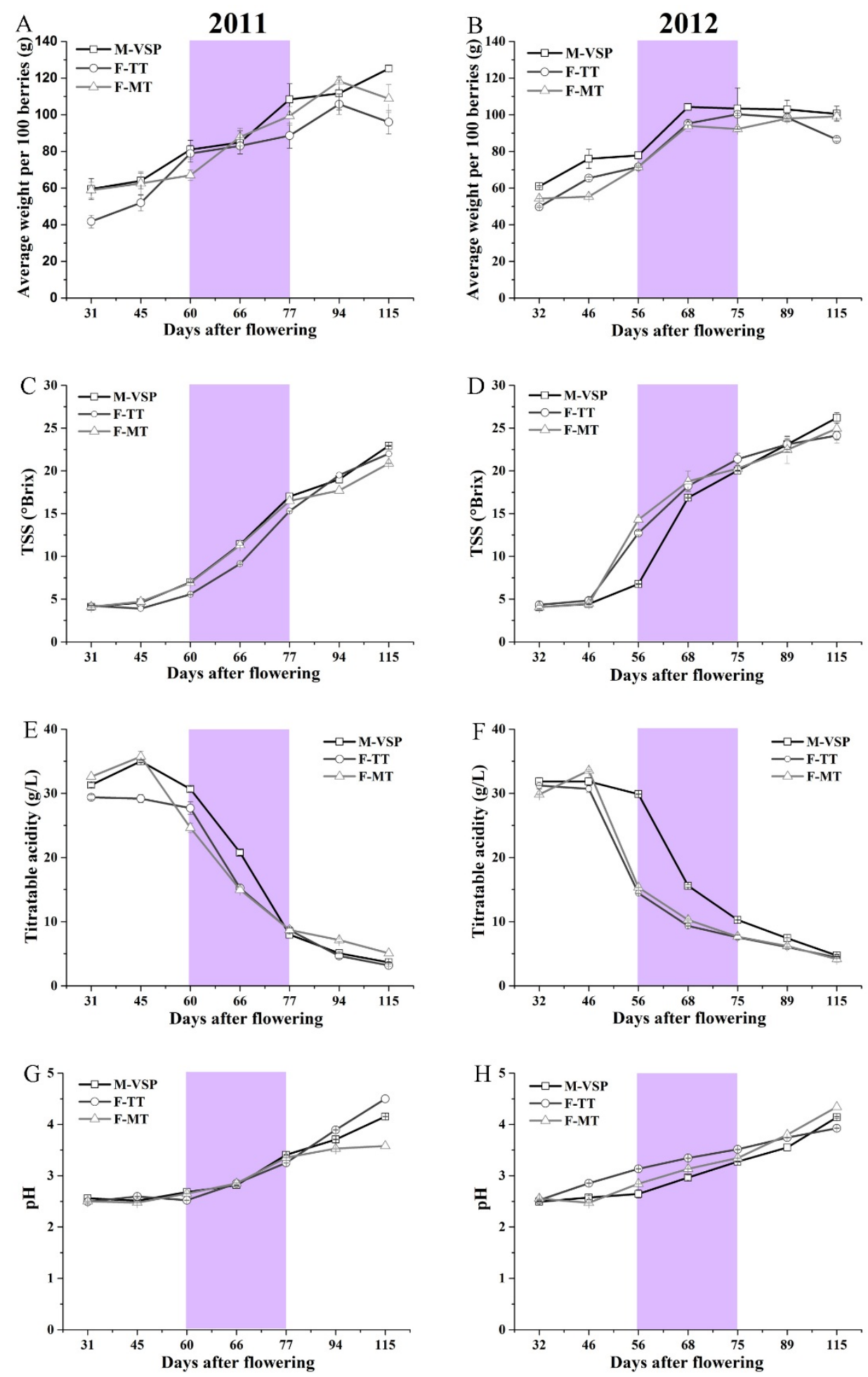

FIGURE 1

Some physical and chemical analyses of Cabernet Sauvignon grapes during development in 2011 (left) and 2012 (right).

point of $\mathrm{pH} 8.2$ and expressed as tartaric acid equivalents. Each analysis was carried out in triplicate for the seed samples.

Climatic data of the region was obtained from the China Meteorological Data Sharing Service System (http://cdc. cma.gov.cn/home.do). The accumulated heat was expressed as growing degree days (GDD, calculated from daily mean temperature, base $10^{\circ} \mathrm{C}$ ).
Extraction of flavan-3-ols in grape seeds

Three replicates of grape samples (50 berries per replicate) were selected for each developmental stage. The grapes were counted and defrosted, and the seeds were separated from the pulp. Berry seeds were frozen, crushed and then freeze-dried. Both the wet and dry weights of the seeds were recorded.

The extraction procedure was performed as described by Liang et al. (2012), with some modifications. For flavan-3-ol 
monomers, $0.1 \mathrm{~g}$ of the lyophilised seed powder was mixed with $1 \mathrm{~mL}$ extraction solution (acetone/distilled $\mathrm{H}_{2} \mathrm{O}, 7: 3$ $\mathrm{v} / \mathrm{v}$ ) in a screw-top $5 \mathrm{~mL}$ Eppendorf tube. In order to prevent oxidation, $0.005 \mathrm{~g}$ ascorbic acid was added. The mixture was centrifuged for $15 \mathrm{~min}\left(8000 \mathrm{~g}, 4^{\circ} \mathrm{C}\right)$, after which the supernatant was collected. The residue was extracted twice in the same way, and the supernatants from three extractions were mixed. A total of $400 \mu \mathrm{L}$ of supernatant was transferred to new tubes and dried rapidly with a stream of dry nitrogen at $30^{\circ} \mathrm{C}$. The dried samples were dissolved in $200 \mu \mathrm{L}$ of methanol acidified with $1 \% \mathrm{HC} 1$, and then neutralised with $200 \mu \mathrm{L}$ of sodium acetate $(200 \mathrm{mM}, \mathrm{pH} 7.5)$. For the proanthocyanidins, $0.1 \mathrm{~g}$ of the lyophilised seed powder was mixed with $1 \mathrm{~mL}$ phloroglucinol buffer $(0.25 \mathrm{~g}$ ascorbate, $1.25 \mathrm{~g}$ phloroglucinol, $215 \mathrm{~mL}$ conc. $\mathrm{HCl}, 25 \mathrm{~mL}$ methanol) and incubated at $50^{\circ} \mathrm{C}$ for $20 \mathrm{~min}$, then neutralised with $1 \mathrm{~mL}$ of sodium acetate $(200 \mathrm{mM}, \mathrm{pH} 7.5)$ to terminate the reaction. The reaction liquid was centrifuged for $15 \mathrm{~min}$ $\left(10000 \mathrm{~g}, 4^{\circ} \mathrm{C}\right)$. The residue was extracted twice using the same method, and the supernatants were pooled. Three independent extractions were done for each sampling date.

\section{Analysis of flavan-3-ols by HPLC-DAD-ESI-MS/MS}

The extracts obtained above were filtered through $0.22 \mu \mathrm{m}$ inorganic membranes (Membrana Co., West Germany) and then analysed directly. The analyses were performed by an Agilent 1200 Series HPLC-DAD-ESI-MS/MS, using a reverse-phase column (Zorbax SB-C18, 250×4.6 mm, $5 \mu \mathrm{m})$, and MS analysis was done in the negative ionisation mode. The column temperature was maintained at $25^{\circ} \mathrm{C}$. Two solvents with a flow rate of $1 \mathrm{~mL} / \mathrm{min}$ were used: $0.2 \%$ $\mathrm{v} / \mathrm{v}$ aqueous acetic acid (mobile phase A) and acetonitrile -
$0.2 \%$ acetic acid $(4: 1 \mathrm{v} / \mathrm{v})($ mobile phase $\mathrm{B})$. The gradients of solvent $\mathrm{B}$ was as follows: $0 \mathrm{~min}, 10 \% ; 20 \mathrm{~min}, 10 \% ; 30$ $\min , 15 \%$; $40 \mathrm{~min}, 20 \%$; $50 \mathrm{~min}, 33 \%$; $55 \mathrm{~min}, 40 \%$; 58 min, 100\%; $63 \mathrm{~min}, 100 \%$; and $64 \mathrm{~min}, 10 \%$. An injection volume of $25 \mu \mathrm{L}$ was used and the eluent was monitored at $280 \mathrm{~nm}$ using DAD detection. The column was then reequilibrated with $10 \% \mathrm{~B}$ for $5 \mathrm{~min}$ before the next injection. The following parameters were set: nebuliser pressure, 30 psi; dry gas flow, $10 \mathrm{~mL} / \mathrm{min}$; dry gas temperature, $325^{\circ} \mathrm{C}$; scan at $\mathrm{m} / \mathrm{z} 100$ to 1000 .

The flavan-3-ol monomers and phloroglucinol adducts were qualitative and quantitative by using their standard curves and their corresponding negative mode MS information (Table 1). Proanthocyanidin was cleaved by excess phloroglucinol into two parts. The flavan-3ol phloroglucinol adducts were regarded as extension subunits, while the other flavan-3-ol subunits were terminal subunits. The content of terminal subunits was calculated by subtracting the content of the free monomers in the acetoneextraction sample from the total free monomers of the acid-catalysis sample. The mean degree of polymerisation (mDP) was calculated as followed (Kennedy \& Jones, 2001; Downey et al., 2003):

$$
\mathrm{mDP}=\frac{\text { total terminal subunits }+ \text { total extension subunits }}{\text { total terminal subunits }}
$$

\section{Statistical analysis}

Statistical analyses were performed using SPSS 20 software (SPSS Inc., USA). One-way analysis of variance (ANOVA) was applied to all samples to determine the significant difference at the 5\% level. Principal component analysis and two-way analysis of variance were implemented to compare

\section{TABLE 1}

Monthly rainfall, growing degree days, and maximum and minimum and average air temperatures in the 2011 and 2012 vintages

\begin{tabular}{|c|c|c|c|c|c|c|c|c|c|c|}
\hline \multirow[b]{2}{*}{ month } & \multicolumn{5}{|c|}{2011} & \multicolumn{5}{|c|}{2012} \\
\hline & $\begin{array}{l}\text { Rain } \\
(\mathrm{mm})\end{array}$ & $\begin{array}{l}\text { GDD } \\
\left({ }^{\circ} \mathrm{C}\right)\end{array}$ & $\begin{array}{l}\mathbf{T}_{\max } \\
\left({ }^{\circ} \mathrm{C}\right)\end{array}$ & $\begin{array}{l}\mathbf{T}_{\min } \\
\left({ }^{\circ} \mathrm{C}\right)\end{array}$ & $\begin{array}{l}\mathbf{T}_{\mathbf{m}} \\
\left({ }^{\circ} \mathrm{C}\right)\end{array}$ & $\begin{array}{l}\text { Rain } \\
(\mathrm{mm})\end{array}$ & $\begin{array}{l}\text { GDD } \\
\left({ }^{\circ} \mathrm{C}\right)\end{array}$ & $\begin{array}{l}\mathbf{T}_{\max } \\
\left({ }^{\circ} \mathrm{C}\right)\end{array}$ & $\begin{array}{l}\mathbf{T}_{\min } \\
\left({ }^{\circ} \mathrm{C}\right)\end{array}$ & $\begin{array}{l}\mathbf{T}_{\mathbf{m}} \\
\left({ }^{\circ} \mathrm{C}\right)\end{array}$ \\
\hline Jan & 6 & 0 & -14.1 & -27.7 & -21.8 & 5.3 & 0 & -6.9 & -23.9 & -17.4 \\
\hline Feb & 13.8 & 0 & -3.1 & -18.2 & -8.8 & 9 & 0 & -4.5 & -17.9 & -12.6 \\
\hline March & 10.1 & 0 & 8.4 & -17.4 & -5.1 & 13.5 & 1 & 11 & -11.1 & 1.3 \\
\hline April & 25.4 & 190.8 & 23 & 0.4 & 15.4 & 9.3 & 188.2 & 22.5 & 9 & 16.3 \\
\hline May & 36.7 & 296.5 & 27.8 & 13.2 & 19.6 & 9.3 & 350.9 & 27.5 & 13 & 21.3 \\
\hline June & 28.3 & 473.1 & 28.8 & 20.8 & 25.8 & 16 & 497.6 & 31.1 & 23 & 26.6 \\
\hline July & 21.4 & 519.9 & 32.6 & 20.4 & 26.8 & 21.3 & 528.5 & 32.5 & 19.6 & 27 \\
\hline Aug & 41.2 & 462 & 30.4 & 16.1 & 24.9 & 13.4 & 447 & 31.4 & 23.1 & 25.9 \\
\hline Sep & 0 & 278.9 & 24.9 & 13.3 & 19.3 & 15.6 & 276.5 & 27.8 & 10.9 & 19.3 \\
\hline Oct & 27.1 & 69.4 & 18.4 & 0.8 & 10.6 & 18.7 & 37.1 & 15.6 & 4.9 & 9.8 \\
\hline Nov & 4.4 & 0 & 4.8 & -3.5 & 0.8 & 23 & 0 & 6.9 & -12.6 & -3.8 \\
\hline Dec & 3.4 & 0 & 1.3 & -18.6 & -9.9 & 25.4 & 0 & -7 & -26.1 & -15.5 \\
\hline Total & 217.8 & 2290.6 & & & & 179.8 & 2326.8 & & & \\
\hline
\end{tabular}

Notes: GDD, growing degree days; $\mathrm{T}_{\max }$, maximum air temperature; $\mathrm{T}_{\min }$, minimum air temperature; $\mathrm{T}_{\mathrm{m}}$, average air temperature 
the effect of vintages and training system on the content and composition of flavan-3-ols in the harvested grape seeds. Each data point, expressed as milligram equivalent of the respective compound per berry, was the average of the data including three extraction replicates.

\section{RESULTS AND DISCUSSION \\ Climatic conditions}

Differences in temperature and rainfall were observed in the study area between the 2011 and 2012 vintages (Table 1). During grape development, most of maximum, minimum and average monthly temperatures were higher for the 2012 than the 2011 vintage. The observed higher temperature in the 2012 vintage helped accumulate heat for grape growth. In addition, higher total rainfall was found in the 2011 vintage, which can be attributed to the occurrence of a large amount of rainfall in August. No rain fell during grape ripening in 2011, while there was some rainfall in 2012. High rainfall is likely to decrease grape maturity and increase the incidence of grape diseases. A mathematical formula was used for calculating GDD, and the results showed that cumulative growing degree days was 2290.6 GDD in 2011, lower than the 2326.8 GDD in 2012. The displayed seasonal variability shows that 2012 was a drier and warmer vintage compared to 2011 .

\section{General analytical parameters}

The average weight of 100 berries during grape development showed that M-VSP accelerated berry growth compared to that of F-TT and F-MT in both vintages (Fig. $1 \mathrm{~A}$ and B). As a result, M-VSP showed the highest average weight of 100 berries at harvest. During véraison, F-TT showed the slowest sugar accumulation in 2011, but in 2012 the slowest sugar accumulation was found in M-VSP berries. However, after véraison, M-VSP enhanced sugar accumulation, causing a higher TSS at harvest (Fig. $1 \mathrm{C}$ and D). As for the parameter of titratable acidity, M-VSP showed a decline, but reached similar values at harvest to the other two training systems (Fig. $1 \mathrm{E}$ and $\mathrm{F}$ ). The $\mathrm{pH}$ of the must was similar among the three training systems, with the highest value obtained for the F-TT harvested grape must from the 2011 vintage; M-VSP presented slightly lower $\mathrm{pH}$ values during grape development in 2012 (Fig. $1 \mathrm{G}$ and H). In addition, the 2012 vintage showed higher values of TSS and lower values of average weight per 100 berries than the 2011 vintage.

\section{Effect of training system on the composition of flavan- 3-ols}

The content of total flavan-3-ols extracted from Cabernet Sauvignon grape seeds during the 2011 and 2012 vintages was calculated as mg/berry (Fig. 2 A and B). Some common patterns of total flavan-3-ol accumulation can be observed for the three training systems in both vintages, which exhibited an increase followed by a decline. The observed pattern was consistent with previous studies (Romeyer et al., 1985; Katalinić \& Maleš, 1997; Kennedy et al., 2000a, 2000b; Giovanelli \& Brenna, 2007). However, the highest total flavan-3-ol contents were found at different DAF among the three training systems in both vintages. During the 2011 vintage, the highest contents of total flavan-3-ols for M-VSP and F-TT were observed at the beginning of véraison (5.54 and $5.71 \mathrm{mg} /$ berry respectively), whereas F-MT presented the highest value earlier, at 45 DAF (4.29 mg/berry). During the 2012 vintage, the highest content of flavan-3-ols for M-VSP, F-TT and F-MT occurred at 56, 46 and 68 DAF respectively, with values of $5.46,4.19$ and $4.21 \mathrm{mg} /$ berry respectively. The decline of total flavan-3-ols was possibly due to the lower extraction from seeds with maturity (Riou et al., 2002; Hanlin et al., 2010). Other research has suggested that this decline can be attributed to oxidative degradation (Geny et al., 2003), because the grape seeds experienced an oxidative process during grape ripening (Kennedy et al., 2000a).

Compared with other training systems, M-VSP consistently maintained a relatively higher flavan-3-ol content during the grape developmental stages in both vintages. The content of total flavan-3-ols in harvested grape seeds was $3.10 \mathrm{mg} /$ berry in 2011, which exhibited significantly higher levels of $24.5 \%$ and $37.4 \%$ than F-TT and F-MT respectively $(p \leq 0.05)$. However, no significant difference was found between M-VSP (3.48 mg/berry), F-TT ( $3.22 \mathrm{mg} /$ berry) and F-MT (3.30 mg/berry) during the 2012 vintage. The higher content of total flavan-3-ols observed in the M-VSP vines could be attributed to their higher vigour, evidenced in the results of Xu et al. (2015), who found that the M-VSP system could greatly promote light interception and photosynthetic efficiency. Furthermore, differences in the content of total flavan-3-ols were also observed between the two vintages. The total flavan-3-ols were higher in 2011 than in 2012 from fruit set to véraison, while the decrease was especially marked during ripening in the 2011 vintage, thus similar or even lower values were found during grape ripening in the 2011 vintage. Before véraison, the observed higher levels of total flavan-3-ols in 2011 was mainly due to their higher initial levels. In fact, a greater synthesis of flavan-3-ols was found in 2012 during this period. The different behaviour between the 2011 and 2012 vintages could be explained by the differences in temperature and rainfall. Previous authors have indicated that sun exposure might differentially affect the biosynthesis of flavan-3-ols in grape seeds. Adequate light significantly increases the accumulation and expression of biosynthetic genes (Downey et al., 2004). Conversely, extreme temperature $\left(>35^{\circ} \mathrm{C}\right)$ has a negative influence (Jones, 2013). The climatic conditions showed that 2012 was a warmer and drier year, with longer sunlight duration, which favoured the accumulation of flavan-3-ols.

Three flavan-3-ols units were detected in the grape seeds: C, EC and ECG. ECG represented the major component during the developmental stages, varying from 0.7 to $2.7 \mathrm{mg} /$ berry (Fig. $2 \mathrm{C}$ to $\mathrm{H}$ ). The followed component was $\mathrm{EC}$, ranging from 0.7 to $2.2 \mathrm{mg} /$ berry. The lowest content was found for $\mathrm{C}$, which was less than $1 \mathrm{mg} /$ berry. The three observed flavan-3-ol units are typical of grape seed tannins, and this is in accordance with a previous study (Prieur et al., 1994). Prieur et al. (1994) demonstrated that the EC, C, and ECG were in decreasing order. Generally, the evolution of the contents of these three flavan-3-ols units was similar to that of total flavan-3-ols, showing few differences. Taking into account the differences, the effect of training system on 
the content of EC in both vintages was exaggerated, leading to the occurrence of some significant differences $(p \leq 0.05)$. For example, the content of EC in harvested grape seeds from M-VSP was significantly higher, by $31.0 \%$, than that from
F-TT ( $1.58 \mathrm{mg} /$ berry compared to 1.09 for F-TT) during the 2011 vintage $(p \leq 0.05)$, and its value in 2012 was $1.73,1.43$ and $1.59 \mathrm{mg}$ /berry for M-VSP, F-TT and F-MT respectively, also at significant levels $(p \leq 0.05)$. This difference indicated
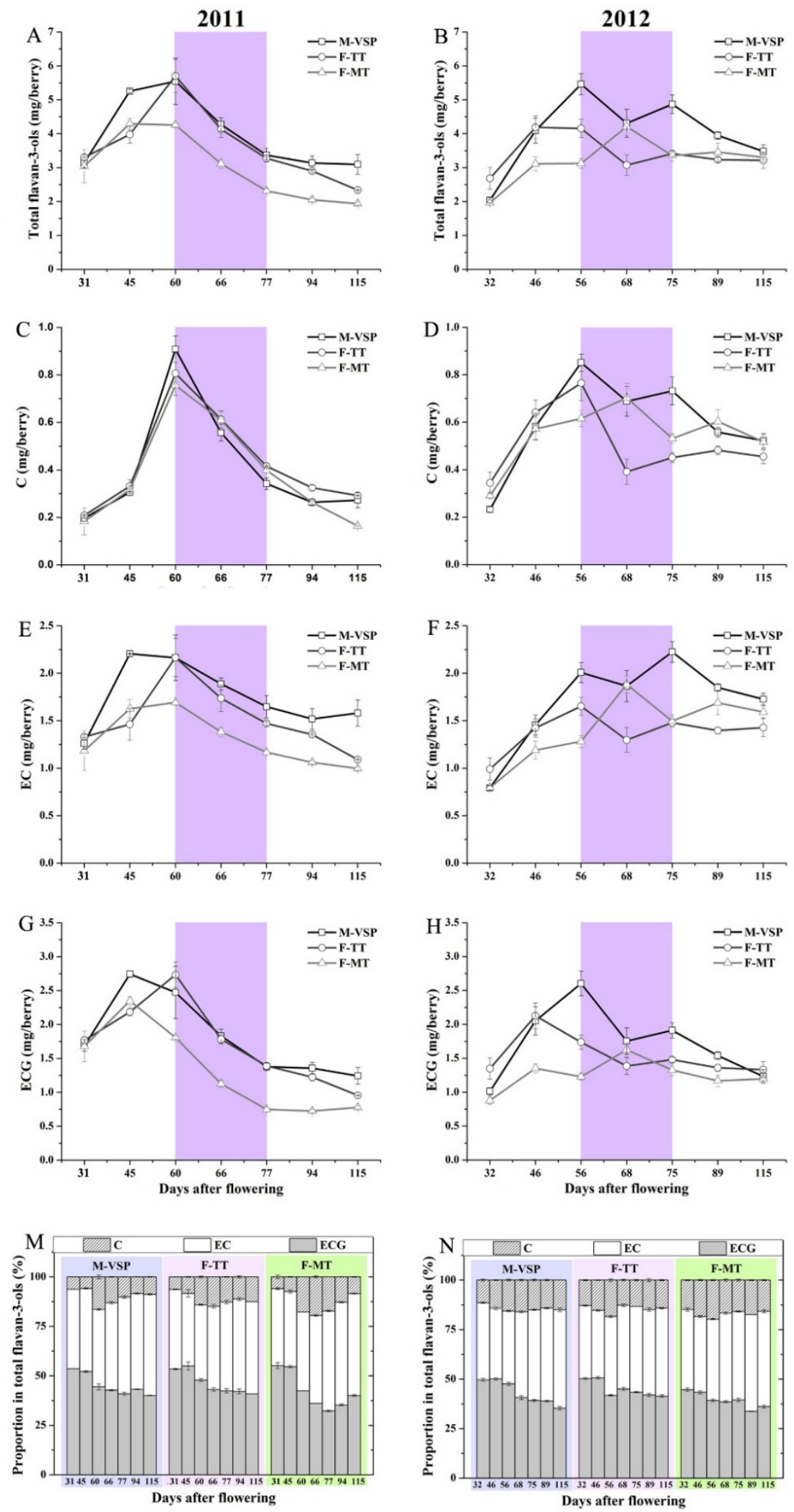

FIGURE 2

The content and composition of flavan-3-ol accumulation in Cabernet Sauvignon grape seeds during development in 2011 (left) and 2012 (right). The values are expressed as $\mathrm{mg} /$ berry and \% proportion. 
that M-VSP was more conducive to accumulating EC compared with the other training systems, especially in a relatively wet year (2011). C, in contrast, showed a relatively lower content in the harvested grape seeds from M-VSP in both vintages. As for the content of ECG, the lower value for M-VSP was only found in the 2012 vintage. In fact, $C$ and EC were both biosynthesised through the flavonoid pathway (Fig. 2), which shared the same branch of the pathway (Koyama et al., 2012). Hence the observed differences were probably a main effect of M-VSP due to the higher vigour favouring the synthesis of EC.

The proportions of the three flavan-3-ol units in the grape seeds are shown in Fig. 2 ( $\mathrm{M}$ and $\mathrm{N}$ ). The flavan-3-ol units of EC and ECG represent the major components during the developmental stages, accounting for $30 \%$ to $55 \%$, while $\mathrm{C}$ represented $5 \%$ to $20 \%$. During the 2011 vintage, the proportion of EC presented an increasing trend with some fluctuations, while ECG showed the opposite trend. As for the evolution of $\mathrm{C}$, the proportion increased from fruit set to véraison, followed by a decline. The 2012 vintage showed a similar evolution of the proportions of these three flavan-3-ol units. However, because the increase in the proportion of $\mathrm{C}$ was more intensive in the 2011 vintage, a lower proportion of $\mathrm{C}$ was only found at véraison in the 2012 vintage. Accordingly, the 2011 vintage showed higher proportions of EC and ECG. Considering the training systems, their effect on the evolution of the proportions of the three flavan-3ol units was not noticeable in both vintages. In addition, a great decrease in the proportion of ECG occurred in M-VSP, and its value in the harvested grape seeds was low, which potentially might reduce the perception of coarseness in the resulting wines (Vidal et al., 2003).

\section{Effect of training system on the composition of terminal subunits}

The evolution of the levels of terminal subunits in the seeds is shown in Fig. $3 \mathrm{~A}$ and B. The content of terminal subunits exhibited an increase from fruit set to véraison, followed by a significant decrease for the three training systems in the 2011 vintage $(p \leq 0.05)$. However, the evolution of the levels of terminal subunits in 2012 was different from that of 2011, showing a gradual increase from fruit set to harvest, with some fluctuations. The effect of training system on the content of terminal subunits varied between the 2011 and 2012 vintages. During the 2011 vintage, the highest value of terminal subunits for F-MT $(2.16 \mathrm{mg} /$ berry $)$ showed a significantly lower value for M-VSP and F-TT, by $25.5 \%$ and $14.3 \%$ respectively $(p \leq 0.05)$. At harvest, their levels decreased by $75.5 \%, 63.8 \%$ and $71.7 \%$, respectively, displaying significant differences among them $(p \leq 0.05)$. From these results it can be seen that M-VSP presented a relatively higher content of terminal subunits compared with the two other training systems. In 2012, the content of terminal subunits was less than $1.2 \mathrm{mg} /$ berry. Their values displayed little difference in the harvested grape seeds.

The terminal subunits detected in the grape seeds were $\mathrm{C}, \mathrm{EC}$ and ECG, which was the same as the flavan-3-ol units (Fig. $3 \mathrm{C}$ and D). Among the three terminal subunits, ECG was the dominant component, followed by EC and C. This result is inconsistent with previous research (Prieur et al., 1994).
The observed variation in the major terminal subunit could be determined by vineyard management, site, cultivar and vintage. For a given vintage, the evolution of the proportion of the three terminal subunits showed similar patterns to that of the flavan-3-ol units. For example, the proportion of the ECG terminal subunit decreased from $77 \%$ at fruit set to $45 \%$ at harvest in the 2011 vintage, while the proportion of the EC terminal subunit increased from $5 \%$ to $35 \%$ in the same period. Regarding the proportion of the $\mathrm{C}$ terminal subunit, its level fluctuated around $20 \%$. Considering the effect of the vintages, the proportion of $\mathrm{EC}$ and $\mathrm{C}$ terminal subunits was higher in 2011 than in 2012. Another obvious difference was that M-VSP presented a relatively higher proportion of EC terminal subunits compared with F-TT and F-MT in both vintages.

\section{Effect of training system on the composition of extension subunits}

The evolution of the levels of extension subunits demonstrated consistent treatment and vintage effects with that of terminal subunits (Fig. $3 \mathrm{E}$ and F). The extension subunits for M-VSP, F-TT and F-MT in 2011 increased from fruit set to 45, 60 and 45 DAF respectively, followed by a decrease. Compared with the 2011 vintage, the content of extension subunits in the 2012 vintage tended to increase from fruit set to harvest, with some fluctuations, resulting in a significantly higher content of extension subunits in the harvested grape seeds $(p \leq 0.05)$. At harvest, the contents of extension subunits for M-VSP in 2011 were $21.5 \%$ lower than the samples in 2012 (2.05 vs. $2.49 \mathrm{mg} /$ berry), 50\% lower for F-TT (1.56 vs. $2.34 \mathrm{mg} /$ berry) and $81.5 \%$ lower for F-MT (1.30 vs. $2.36 \mathrm{mg} /$ berry). Taking into account the differences among the three training systems, it can be seen that M-VSP presented a higher content of extension subunits in both vintages, which might be correlated with the observed higher content of total flavan-3-ols for M-VSP.

The abovementioned three units of C, EC and ECG found in the terminal subunits were also detected in the extension subunits (Fig. $3 \mathrm{G}$ and $\mathrm{H}$ ), but ECG was not dominant in extension subunits. The EC extension subunit accounted for $45 \%$ to $68 \%$, followed by the proportion of the ECG extension subunit (from $25 \%$ to $48 \%$ ). The lowest proportion was for the $\mathrm{C}$ extension subunit (from $2 \%$ to $15 \%$ ). The observed result is in agreement with previous studies (Prieur et al., 1994; Kennedy et al., 2000b; Hanlin et al., 2011). In 2011, the proportion of ECG extension subunits decreased, while the EC extension subunits increased in proportion, and the proportion of the $\mathrm{C}$ extension subunit was less than $5 \%$. In 2012, little variation was observed in the evolution of the proportions of the three extension subunits, with ECG, $\mathrm{EC}$ and $\mathrm{C}$ remaining in their proportions of around 35\%, $55 \%$ and $10 \%$ respectively. In addition, there were some differences in the levels of the proportions between the 2011 and 2012 vintages. Compared with 2011 vintage, the 2012 vintage showed a significantly higher proportion of $\mathrm{C}$ in the extension subunits, indicating that the higher GDD and lower rainfall contributed to the formation of the $\mathrm{C}$ extension subunit. As for the effect of training system, F-MT presented a higher proportion of EC in extension subunits during grape ripening in both vintages. 

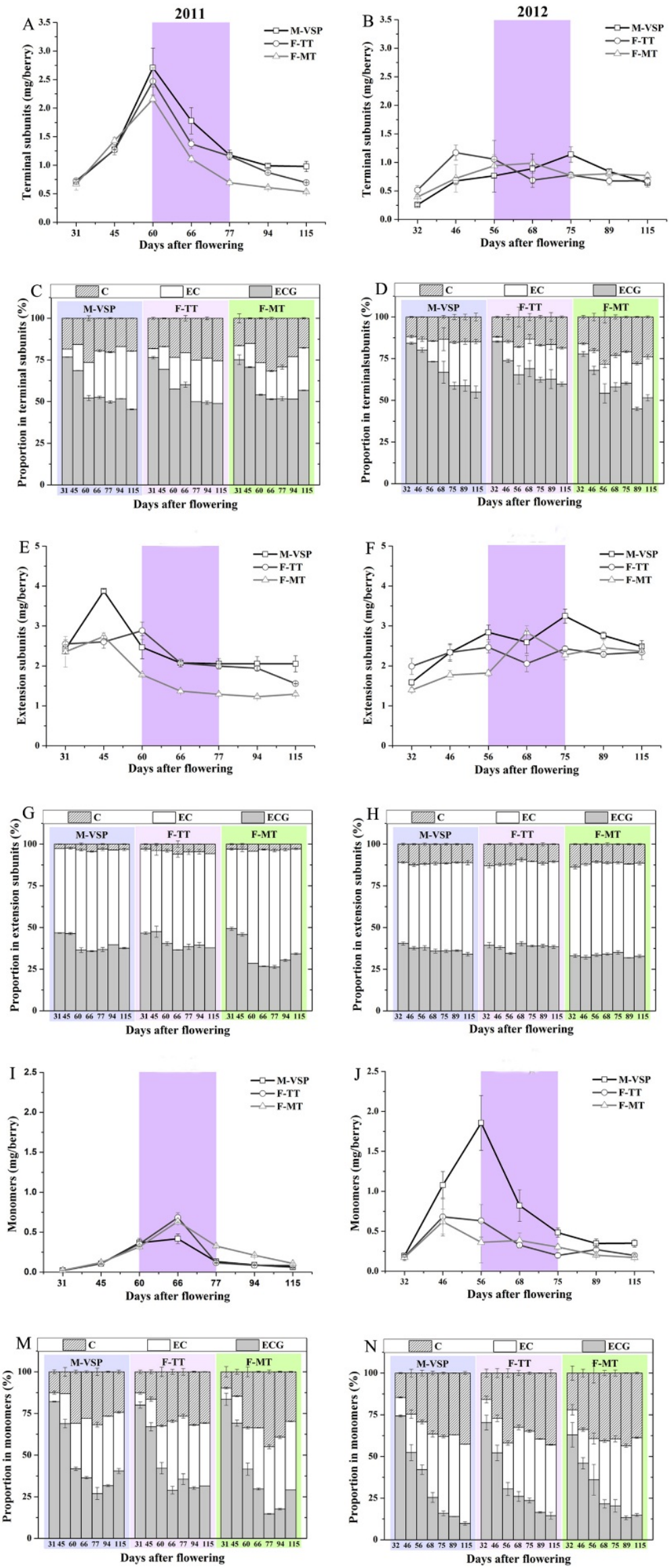

FIGURE 3

The content and composition of terminal subunits, extension subunits and monomers in Cabernet Sauvignon grape seeds during development in 2011 (left) and 2012 (right). The values are expressed as $\mathrm{mg} /$ berry and \% proportion. 
Effect of training system on the composition of monomers The evolution of the levels of monomers was similar in both vintages, which exhibited an increase followed by a decline (Fig. $3 \mathrm{I}$ and J). This pattern of monomer accumulation was consistent with that of a previous study (Ristic \& Iland, 2005). The content of monomers for M-VSP, F-TT and F-MT reached their highest values at $66 \mathrm{DAF}$ (with values of 0.42 , 0.68 and $0.64 \mathrm{mg} /$ berry respectively) in 2011, for which M-VSP presented a significantly lower level $(p \leq 0.05)$. This lower value found in M-VSP was not consistent with the total flavan-3-ols, suggesting that the formation of proanthocyanidin originating from monomers was more accessible for M-VSP. However, the content of monomers in harvested grape seeds showed no significant difference among the three training systems. Unlike the 2011 vintage, M-VSP displayed the highest content of monomers during the grape developmental stages in the 2012 vintage. For example, its highest content was $1.86 \mathrm{mg} /$ berry at $56 \mathrm{DAF}$, whereas $0.63 \mathrm{mg} /$ berry was presented in F-TT at the same time, which is almost three times as high as that of F-TT. As mentioned above, the harvested grape seeds in 2012 had a higher monomer content in comparison with 2011.

$\mathrm{C}, \mathrm{EC}$ and ECG were all detected in the monomers. In the early period of grape development, the main monomer component was ECG. During grape ripening, none of the three could be regarded as the main component (Fig. $3 \mathrm{M}$ and $\mathrm{N}$ ). The proportion of ECG in the monomers decreased (from $84 \%$ to $9 \%$ ), while the proportions of $\mathrm{C}$ and $\mathrm{EC}$ in increased (from $10 \%$ to $50 \%$ and from $9 \%$ to $45 \%$ respectively). A higher proportion of ECG in monomers was found in 2011, and its value fluctuated during grape ripening. There were some other differences in the proportions among the three training systems in both vintages, but the differences were not significant.

\section{Effect of training systems on the value of $\mathrm{mDP}$}

The values of $\mathrm{mDP}$ in grape seeds varied throughout berry development (Fig. 4 A and B). During the 2011 vintage, the $\mathrm{mDP}$ for F-TT and F-MT reached its highest value at véraison, after which it decreased. In contrast, the evolution of $\mathrm{mDP}$ for M-VSP showed the opposite trend, reaching its lowest level at véraison. The lowest $\mathrm{mDP}$ value in M-VSP at véraison might be attributable to the greater magnitude of the conversion between monomers and terminal subunits, as observed by Kennedy et al. (2000b). During the 2012 vintage, the evolution of $\mathrm{mDP}$ in F-TT and F-MT decreased slightly from fruit set to véraison, followed by a slight increase, while M-VSP showed a declining tendency, with some fluctuations. From these results it can be seen that the evolution of $\mathrm{mDP}$ varies between training systems and vintages. A previous study suggest that $\mathrm{mDP}$ decreases as grapes ripen (Kennedy et al., 2000b), while Downey et al. (2003) show that the mDP varies without obvious trend during grape ripening. The value of $\mathrm{mDP}$ ranged from two subunits to six subunits, which is consistent with previous work (Cheynier et al., 1992; Chira et al., 2009). However, the study by Bordiga et al. (2011), which was done in a cooler growing regions than the current study, shows a higher value of $\mathrm{mDP}$ (above 10 subunits) than the current study. This difference is possibly due to regional and environmental differences (Hanlin \& Downey, 2009). As for the value of $\mathrm{mDP}$ in harvested grape seeds, M-VSP exhibited a significantly higher value compared with F-TT and F-MT.

\section{Principal component analysis (PCA)}

To have an overview of the effect of training system on the content and composition of flavan-3-ols, the above-mentioned parameters in harvested grape seeds were subjected to a PCA (Fig. 5 A). The parameters included the content of total flavan-3-ols (Fla), terminal subunits (Ter), extension subunits (Ext), monomers (Mon), C, EC and ECG, and mDP. Various ratios, like the ratio of the three units to their corresponding subunits, were also included (C/Fla, EC/Fla, ECG/Fla, C/ Ter, EC/Ter, ECG/Ter, C/Ext, EC/Ext, ECG/Ext). Generally, the effect of training system on the parameters differentiated between the 2011 and 2012 vintages. The first two principal components explained $72.39 \%$ of the total variance in the grape seeds samples, with $53.85 \%$ and $18.55 \%$ explained by $\mathrm{PC} 1$ and $\mathrm{PC} 2$ respectively. PC1 was strongly correlated with $\mathrm{mDP}$ values, Fla, C, EC, ECG, Mon, Ext and EC/Mon, which were located on the positive side of $\mathrm{PC} 1$. The negative side of PC1 corresponded to ECG/Fla, ECG/Mon and EC/ Ext. PC2 was strongly correlated with Ter, EC/Ter and ECG/ Ext. EC/Ter was located on the positive side of $\mathrm{PC} 2$, while $\mathrm{ECG} /$ Ter was on the negative side, indicating that the EC/ Ter mainly increased along with the decline in ECG/Ter (Fig. 2). Fig. $5 \mathrm{~B}$ shows the corresponding score scatterplot to separate the grape seed samples. PC1 led to the separation of samples from different vintages, with the samples from 2012 being on the positive side of PC1. From these results it can be concluded that mDP, Fla, Ext, Mon, C, EC and ECG were higher in the 2012 vintage, while ECG/Fla, ECG/Mon and EC/Ext were higher in the 2011 vintage. PC2 discriminated between samples trained on the three systems (M-VSP, F-TT and F-MT). M-VSP was located on the positive side of PC2, while F-TT was located on the negative side. In addition, the efficiency of the 2011 vintages on the different training systems was more obvious than that of 2012. This indicated that in a wet vintage, such as 2011, it is much easier to discriminate between the three training systems. The fact that, in 2012, M-VSP was in the first quadrant, indicates that M-VSP contributed to the levels of Fla, Ext, C/Fla, EC/Fla, $\mathrm{EC} /$ Ext and EC/Mon in the 2012 vintage.

The parameters of the content and composition of flavan3-ols in harvested grape seeds in the two vintages of study are shown in Table 2, which illustrates a two-way ANOVA using training system and vintage as factors. Among the 20 parameters analysed in this study, 19 exhibited significantly different levels, depending on the main effect of vintages or training systems, and 18 were significantly affected by the interaction effects of both factors. Of those parameters, the content of terminal subunits was not affected by vintage, whereas the value of $\mathrm{mDP}$ was not affected by training system. The observed phenomenon was similar to the result of previous research, namely that the polymerisation is not reliant on canopy management (Kemp et al., 2011). Furthermore, from the $p$ values it can be concluded that the content of flavan-3-ols and their composition were influenced more by vintage than by training system. This result is consistent with previous research (Xing et al., 2015). 
TABLE 2

Probability values for the effects of both vintages and training systems on the content and composition of flavan-3-ols in Cabernet Sauvignon grape seeds in 2011 and 2012 vintages

\begin{tabular}{|c|c|c|c|}
\hline \multirow[b]{2}{*}{ Parameters } & \multicolumn{3}{|c|}{$p$ value $^{a}$ for factor } \\
\hline & vintages & training systems & Vintages $\times$ training systems $s^{b}$ \\
\hline \multicolumn{4}{|l|}{ Content } \\
\hline total flavan-3-ols & $<0.0001$ & 0.001 & 0.009 \\
\hline $\mathrm{C}$ & $<0.0001$ & 0.025 & 0.001 \\
\hline $\mathrm{EC}$ & $<0.0001$ & $<0.0001$ & 0.005 \\
\hline ECG & $<0.0001$ & 0.005 & 0.007 \\
\hline extension subunits & $<0.0001$ & 0.002 & 0.016 \\
\hline terminal subunits & 0.241 & 0.006 & $<0.0001$ \\
\hline monomers & $<0.0001$ & 0.005 & $<0.0001$ \\
\hline $\begin{array}{l}\mathrm{mDP} \\
\text { Total flavan-3-ols }\end{array}$ & $<0.0001$ & 0.411 & 0.005 \\
\hline $\mathrm{C} \%$ & $<0.0001$ & $<0.0001$ & $<0.0001$ \\
\hline $\mathrm{EC} \%$ & $<0.0001$ & $<0.0001$ & 0.131 \\
\hline $\begin{array}{l}\mathrm{ECG} \% \\
\text { Extension subunits }\end{array}$ & $<0.0001$ & $<0.0001$ & $<0.0001$ \\
\hline $\mathrm{C} \%$ & $<0.0001$ & $<0.0001$ & $<0.0001$ \\
\hline $\mathrm{EC} \%$ & $<0.0001$ & $<0.0001$ & 0.114 \\
\hline $\begin{array}{l}\mathrm{ECG} \% \\
\text { Terminal subunits }\end{array}$ & 0.009 & $<0.0001$ & 0.011 \\
\hline $\mathrm{C} \%$ & 0.014 & $<0.0001$ & $<0.0001$ \\
\hline $\mathrm{EC} \%$ & $<0.0001$ & $<0.0001$ & 0.028 \\
\hline $\begin{array}{l}\text { ECG\% } \\
\text { Monomers }\end{array}$ & 0.001 & 0.019 & $<0.0001$ \\
\hline $\mathrm{C} \%$ & $<0.0001$ & 0.003 & $<0.0001$ \\
\hline $\mathrm{EC} \%$ & $<0.0001$ & $<0.0001$ & $<0.0001$ \\
\hline ECG\% & $<0.0001$ & 0.01 & $<0.0001$ \\
\hline
\end{tabular}

Notes: ${ }^{a}$ Considered to be significant when $p \leq 0.05 .{ }^{b}$ Interaction effect between years and training systems. C: catechin; EC: epicatechin; ECG: epicatechin-3-O-gallate. C\%: the proportion of catechin; EC\%: the proportion of epicatechin; ECG\%: the proportion of epicatechin3-O-gallate
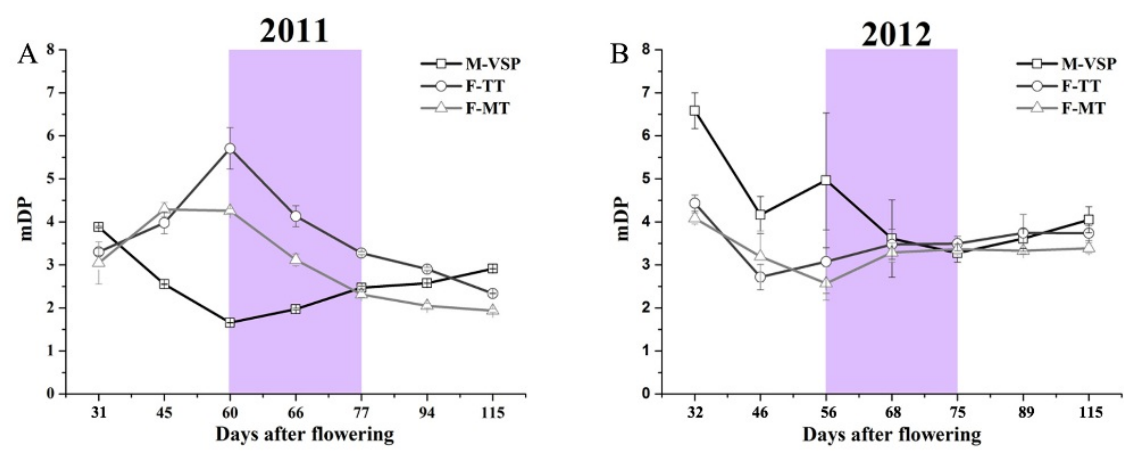

FIGURE 4

The mean degree of polymerisation (mDP) of flavan-3-ols in Cabernet Sauvignon grape seeds during development in 2011 (left) and 2012 (right). 

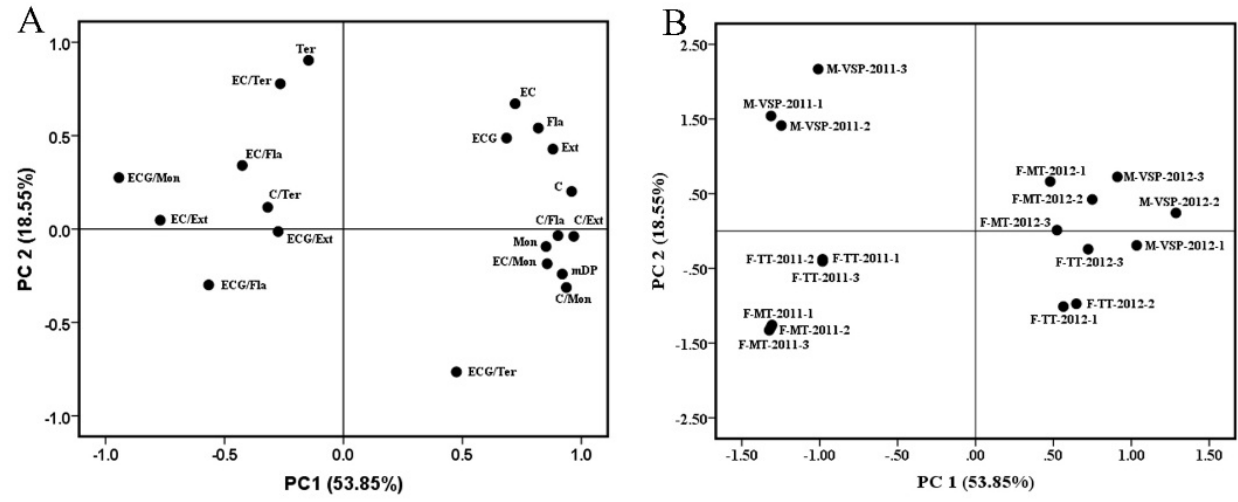

FIGURE 5

Principal component analysis based on different parameters of flavan-3-ols in harvested grape seeds. (A) loading plot; (B) score scatter plot.

\section{CONCLUSIONS}

The present results clearly reveal the content and composition of flavan-3-ols in seeds during the development of Cabernet Sauvignon grapes. There was variation in the content and composition of flavan-3-ols between training systems and vintages, and the extent of this variability was affected more by vintage than by training system. Vintage influenced the effect of training system on flavan-3-ol accumulation, and the relatively wet vintage (2011) could discriminate between the three training systems relatively well. However, the dry vintage (2012) was more beneficial for the accumulation and polymerisation of flavan-3-ols in the harvested grape seeds. Comparing the three training systems, M-VSP contributed the highest content of total flavan-3-ols in the harvested grape seeds in both vintages, followed by F-MT in the 2011 vintage. In the dry vintage of 2012, it was followed by F-TT. In addition, F-TT contributed around $40 \%$ of ECG to total flavan-3-ols, as well as to the extension subunits that represent the majority of tannin polymers in both vintages the highest percentage of the three training systems. From a winemaking perspective, the harvested grape seeds from the M-VSP training system had a higher content of total flavan3-ols and a lower percentage of ECG, which represents positive characteristics that will confer better mouthfeel to the corresponding wine in the future, thus M-VSP may be the best training system among the three training systems in the northwest of China. However, further research is required to determine how the variation in flavan-3-ol composition affects its extraction and the mouthfeel of the wine in order to produce grapes meeting winery specifications.

\section{LITERATURE CITED}

Alcalde-Eon, C., Escribano-Bailón, M.T., Santos-Buelga, C. \& RivasGonzalo, J.C., 2006. Changes in the detailed pigment composition of red wine during maturity and ageing: A comprehensive study. Anal. Chim. Acta 563(1), 238-254.

Aron, P.M. \& Kennedy, J.A., 2008. Flavan-3-ols: Nature, occurrence and biological activity. Mol. Nutr. Food Res. 52(1), 79-104.
Bavougian, C.M., Read, P.E., Schlegel, V.L. \& Hanford, K.J. 2013., Canopy light effects in multiple training systems on yield, soluble solids, acidity, phenol and flavonoid concentration of 'Frontenac' grapes. Hort. Technol. 23(1), 86-92.

Bordiga, M., Travaglia, F., Locatelli, M., Coïsson, J.D. \& Arlorio, M., 2011. Characterisation of polymeric skin and seed proanthocyanidins during ripening in six Vitis vinifera L. cv. Food Chem. 127(1), 180-187.

Casassa, L.F. \& Harbertson, J.F., 2014. Extraction, evolution, and sensory impact of phenolic compounds during red wine maceration. Annu. Rev. Food. Sci. Technol. 5, 83-109.

Cheng, G., He, Y.N., Yue, T.X., Wang, J. \& Zhang, Z.W., 2014. Effects of climatic conditions and soil properties on Cabernet Sauvignon berry growth and anthocyanin profiles. Molecules 19(9), 13683-13703.

Cheynier, V., Rigaud, J. \& Da Silva, J.M.R., 1992. Structure of procyanidin oligomers isolated from grape seeds in relation to some of their chemical properties. Plant Polyphenols 59, 281-294.

Chira, K., Schmauch, G., Saucier, C., Fabre, S. \& Teissedre, P.-L., 2009. Grape variety effect on proanthocyanidin composition and sensory perception of skin and seed tannin extracts from Bordeaux wine grapes (Cabernet Sauvignon and Merlot) for two consecutive vintages (2006 and 2007). J. Agr. Food Chem. 57(2), 545-553.

De Freitas, V.A.P. \& Glories, Y., 1999. Concentration and compositional changes of procyanidins in grape seeds and skin of white Vitis vinifera varieties. J. Sci. Food Agric. 79(12), 1601-1606.

Downey, M.O., Harvey, J.S. \& Robinson, S.P., 2003. Analysis of tannins in seeds and skins of Shiraz grapes throughout berry development. Aust. J. Grape Wine Res. 9(1), 15-27.

Downey, M.O., Harvey, J.S. \& Robinson, S.P., 2004. The effect of bunch shading on berry development and flavonoid accumulation in Shiraz grapes. Aust. J. Grape Wine Res. 10(1), 55-73.

Fragasso, M., Antonacci, D., Pati, S., Tufariello, M., Baiano, A., Forleo, L.R., Caputo, A.R. \& Notte, E.L., 2012. Influence of training system on volatile and sensory profiles of Primitivo grapes and wines. Am. J. Enol. Vitic. 63(4), 477-486.

Fulcrand, H., Dueñas, M., Salas, E. \& Cheynier, V., 2006. Phenolic reactions during winemaking and aging. Am. J. Enol. Vitic. 57(3), 289-297.

Geny, L., Saucier, C., Bracco, S., Daviaud, F. \& Glories, Y., 2003. Composition and cellular localization of tannins in grape seeds during maturation. J. Agr. Food Chem. 51(27), 8051-8054. 
Giovanelli, G. \& Brenna, O.V., 2007. Evolution of some phenolic components, carotenoids and chlorophylls during ripening of three Italian grape varieties. Eur. Food Res. Technol. 225(1), 145-150.

Gladstone, E.A. \& Dokoozlian, N.K., 2003. Influence of leaf area density and trellis/training system on the light microclimate within grapevine canopies. Vitis 42(3), 123-131.

González-Neves, G., Gil, G. \& Ferrer, M., 2002. Effect of different vineyard treatments on the phenolic contents in Tannat (Vitis vinifera L.) grapes and their respective wines. Food Sci. Technol. Int. 8(5), 315-321.

Hanlin, R.L. \& Downey, M.O., 2009. Condensed tannin accumulation and composition in skin of Shiraz and Cabernet Sauvignon grapes during berry development. Am. J. Enol. Vitic. 60(1), 13-23.

Hanlin, R., Hrmova, M., Harbertson, J. \& Downey, M., 2010. Review: Condensed tannin and grape cell wall interactions and their impact on tannin extractability into wine. Aust. J. Grape Wine Res. 16(1), 173-188.

Hanlin, R.L., Kelm, M.A., Wilkinson, K.L. \& Downey, M.O., 2011. Detailed characterization of proanthocyanidins in skin, seeds, and wine of Shiraz and Cabernet Sauvignon wine grapes (Vitis vinifera). J. Agr. Food Chem. 59(24), 13265-13276

Haslam, E., 1998. Practical polyphenolics: From structure to molecular recognition and physiological action. Cambridge University Press, London.

Jones, H.G., 2013 (4 $4^{\text {th }}$ ed). Plants and microclimate: A quantitative approach to environmental plant physiology. Cambridge University Press, London.

Katalinić, V. \& Maleš, P., 1997. Compositional changes in grape polyphenols throughout maturation. J. Wine Res. 8(3), 169-177.

Kemp, B.S., Harrison, R. \& Creasy, G.L., 2011. Effect of mechanical leaf removal and its timing on flavan-3-ol composition and concentrations in $\mathrm{Vi}$ tis vinifera L. cv. Pinot Noir wine. Aust. J. Grape Wine Res. 17(2), 270-279.

Kennedy, J.A. \& Jones, G.P., 2001. Analysis of proanthocyanidin cleavage products following acid-catalysis in the presence of excess phloroglucinol. J. Agr. Food Chem. 49(4), 1740-1746.

Kennedy, J.A., Matthews, M.A. \& Waterhouse, A.L., 2000a. Changes in grape seed polyphenols during fruit ripening. Phytochem. 55(1), 77-85.

Kennedy, J.A., Troup, G.J., Pilbrow, J.R., Hutton, D.R., Hewitt, D., Hunter, C.R., Ristic, R., Iland, P.G. \& Jones, G.P., 2000b. Development of seed polyphenols in berries from Vitis vinifera L. cv. Shiraz. Aust. J. Grape Wine Res. 6(3), 244-254.

Koyama, K., Ikeda, H., Poudel, P.R. \& Goto-Yamamoto, N., 2012. Light quality affects flavonoid biosynthesis in young berries of Cabernet Sauvignon grape. Phytochem. 78, 54-64

Liang, N.N., He, F., Pan, Q.H., Wang, J., Reeves, M.J. \& Duan, C.Q., 2014. Optimization of sample preparation and phloroglucinol analysis of Marselan grape skin proanthocyanidins using HPLC-DAD-ESI-MS/MS. S. Afr. J. Enol. Vitic. 33(1), 122-131.

Liu, M.Y., Chi, M., Tang, Y.H., Song, C.Z., Xi, Z.M. \& Zhang, Z.W., 2015. Effect of three training systems on grapes in a wet region of China: Yield, incidence of disease and anthocyanin compositions of Vitis vinifera cv. Cabernet Sauvignon. Molecules 20(10), 18967-18987.

Maury, C., Sarni-Manchado, P. \& Lefebvre, S., 2001. Influence of fining with different molecular weight gelatins on proanthocyanidin composition and perception of wines. Am. J. Enol. Vitic. 52(2), 140-145.

Morrison, J.C. \& Noble, A.C., 1990. The effects of leaf and cluster shading on the composition of Cabernet Sauvignon grapes and on fruit and wine sensory properties. Am. J. Enol. Vitic. 41(3), 193-200.
Ough, C. \& Nagaoka, R., 1984. Effect of cluster thinning and vineyard yields on grape and wine composition and wine quality of Cabernet Sauvignon. Am. J. Enol. Viticult. 35(1), 30-34.

Peleg, H., Gacon, K., Schlich, P. \& Noble, A.C., 1999. Bitterness and astringency of flavan-3-ol monomers, dimers and trimers. J. Sci. Food Agric. 79(8), 1123-1128

Pérez-Lamela, C., García-Falcón, M.S., Simal-Gándara, J. \& OrriolsFernández, I., 2007. Influence of grape variety, vine system and enological treatments on the colour stability of young red wines. Food Chem. 101(2), 601-606.

Prieur, C., Rigaud, J., Cheynier, V. \& Moutounet, M., 1994. Oligomeric and polymeric procyanidins from grape seeds. Phytochem. 36(3), 781-784.

Renaud, S.D. \& De Lorgeril, M., 1992. Wine, alcohol, platelets, and the French paradox for coronary heart disease. The Lancet 339(8808), 1523 1526 .

Reynolds, A.G. \& Heuvel, J.E.V., 2009. Influence of grapevine training systems on vine growth and fruit composition: A review. Am. J. Enol. Vitic. $60(3), 251-268$

Riou, V., Vernhet, A., Doco, T. \& Moutounet, M., 2002. Aggregation of grape seed tannins in model wine - effect of wine polysaccharides. Food Hydrocolloids 16(1), 17-23.

Ristic, R. \& Iland, P.G., 2005. Relationships between seed and berry development of Vitis vinifera L. cv Shiraz: Developmental changes in seed morphology and phenolic composition. Aust. J. Grape Wine Res. 11(1), 43-58.

Romeyer, F.M., Macheix, J.J. \& Sapis, J.C., 1985. Changes and importance of oligomeric procyanidins during maturation of grape seeds. Phytochem. 25(1), 219-221.

Sabbatini, P., Wierba, K., Clearwater, L. \& Howell, G.S., 2015. Impact of training system and pruning severity on yield, fruit composition, and vegetative growth of 'Niagara' grapevines in Michigan. Int. J. Fruit Sci. 15(3), 237-250.

Santos-Buelga, C., Francia-Aricha, E. \& Escribano-Bailón, M., 1995. Comparative flavan-3-ol composition of seeds from different grape varieties. Food Chem. 53(2), 197-201.

Segade, S.R., Vázquez, E.S., Rodríguez, E.I.V. \& Martínez, J.F.R., 2009 Influence of training system on chromatic characteristics and phenolic composition in red wines. Eur. Food Res. Technol. 229(5), 763-770.

Sun, B., Pinto, T., Leandro, M., Ricardo-Da-Silva, J. \& Spranger, M., 1999. Transfer of catechins and proanthocyanidins from solid parts of the grape cluster into wine. Am. J. Enol. Vitic. 50(2), 179-184.

Vidal, S., Francis, L., Guyot, S., Marnet, N., Kwiatkowski, M., Gawel, R., Cheynier, V. \& Waters, E.J., 2003. The mouth-feel properties of grape and apple proanthocyanidins in a wine-like medium. J. Sci. Food Agric. 83(6), 564-573.

Xing, R.R., He, F., Xiao, H.L., Duan, C.Q. \& Pan, Q.H., 2015. Accumulation pattern of flavonoids in Cabernet Sauvignon grapes grown in a lowlatitude and high-altitude region. S. Afr. J. Enol. Vitic. 36(1), 32-43.

Xu, X.Q., Cheng, G., Duan, L.L., Jiang, R., Pan, Q.H., Duan, C.Q. \& Wang, J., 2015. Effect of training systems on fatty acids and their derived volatiles in Cabernet Sauvignon grapes and wines of the north foot of Mt. Tianshan. Food Chem. 181, 198-206.

Zoecklein, B.W., Wolf, T.K., Pelanne, L., Miller, M.K., Birkenmaier, S.S., 2008. Effect of vertical shoot-positioned, Smart-Dyson, and Geneva double-curtain training systems on Viognier grape and wine composition. Am. J. Enol. Vitic. 59, 11-21. 